\title{
Altered spontaneous brain activity patterns in patients with unilateral acute open globe injury using amplitude of low-frequency fluctuation: a functional magnetic resonance imaging study
}

\author{
This article was published in the following Dove Press journal: \\ Neuropsychiatric Disease and Treatment \\ II August 2016 \\ Number of times this article has been viewed
}

\begin{abstract}
Gang Tan, 1,2,* Xin Huang, 1,3,*
Lei Ye,' An-Hua Wu, ${ }^{2}$

Li-Xian He, ${ }^{2}$ Yu-Lin Zhong, '

Nan Jiang,' Fu-Qing Zhou, ${ }^{4}$

Yi Shao'

'Department of Ophthalmology,

The First Affiliated Hospital

of Nanchang University,

Nanchang, Jiangxi, ' 2 epartment

of Ophthalmology, The First Affiliated Hospital of University of South China,

Hengyang, Hunan, ${ }^{3}$ Department of Ophthalmology, The First People's

Hospital of Jiujiang City, Jiujiang,

${ }^{4}$ Department of Radiology, The First

Affiliated Hospital of Nanchang

University, Jiangxi Province Medical

Imaging Research Institute, Nanchang,

Jiangxi, People's Republic of China

*These authors contributed equally to this work
\end{abstract}

Correspondence: Yi Shao

Department of Ophthalmology,

The First Affiliated Hospital of Nanchang

University, No 17, Yongwaizheng Street,

Donghu District, Nanchang 330006,

Jiangxi, People's Republic of China

Tel +86 79l 88692520

Email freebee99@।63.com

Fu-Qing Zhou

Department of Radiology, The First Affiliated Hospital of Nanchang University, No 17, Yongwaizheng Street, Donghu District, Nanchang 330006, Jiangxi, People's Republic of China

Tel +86 79l 8869 5। 32

Email fq.chou@yahoo.com
Objective: The aim of this study was to evaluate altered spontaneous brain activities in patients with unilateral acute open globe injury (OGI) using amplitude of low-frequency fluctuation (ALFF) method and its relationship with their clinical manifestations.

Patients and methods: A total of 18 patients with acute OGI (16 males and two females) and 18 healthy controls (HCs, 16 males and two females) closely matched in age, sex, and education were recruited in this study. The ALFF method was used to evaluate the altered spontaneous brain activities. The relationships between the mean ALFF signal values of different brain regions and the clinical features were evaluated by correlation analysis. Acute OGI patients were distinguished from $\mathrm{HCs}$ by receiver operating characteristic curve.

Results: Compared with HCs, acute OGI patients had significantly higher ALFF values in the left cuneus, left middle cingulum cortex, and bilateral precuneus. Furthermore, the age of OGI patients showed a negative correlation with the ALFF signal value of the left middle cingulum cortex $(r=-0.557, P=0.016)$ and a negative correlation with the mean ALFF signal value of the bilateral precuneus $(r=-0.746, P<0.001)$. The ALFF signal value of the bilateral precuneus was negatively correlated with the duration of OGI $(r=-0.493, P=0.038)$ and positively correlated with the vision acuity of the injured eye $(r=0.583, P=0.011)$.

Conclusion: Acute OGI mainly induces dysfunction in the left cuneus, left middle cingulum cortex, and bilateral precuneus, which may reflect the underlying pathologic mechanisms of abnormal brain activities in OGI patients.

Keywords: ALFF, open globe injury, resting state, spontaneous activity, magnetic resonance imaging

\section{Introduction}

Ocular trauma is a common eye disease with a sharp decline in vision and has become a worldwide public health issue. The prevalence of ocular trauma is 4.9 per 100,000 in the Mediterranean area. ${ }^{1}$ According to a study, ${ }^{2}$ men have higher incidence of ocular injury than women (74\% vs 26\%). Clinically, ocular trauma can be divided into open globe injury (OGI) and closed globe injury. OGI is often accompanied by corneal injury, iris prolapse, ${ }^{3}$ traumatic cataracts, ${ }^{4}$ and retinal detachment. ${ }^{5}$ Currently, surgery is the main treatment for OGI. ${ }^{6}$ Even with surgical suture, OGI causes severe damages to vision and even blindness in some circumstances.

By detecting retinal detachment, B-scan ultrasonography is an important clinical test for the diagnosis of OGI. ${ }^{7}$ In addition, computed tomography (CT) is another useful 
radiological technology to detect OGI. ${ }^{8}$ Multidetector CT could detect the uncertainties of globe injuries. ${ }^{9}$ Meanwhile, MRI has also been used to evaluate globe injuries. ${ }^{10}$ However, the above mentioned studies focused only on the ocular trauma, whereas other parts of the visual system are often overlooked, including the connecting pathways and the visual cortex. Clinically, in some acute OGI patients with emergent surgery, the ocular trauma was fully recovered, yet the vision still declined. Thus, the mechanisms of dysfunction of brain functional area caused by OGI remain to be further inspected.

Resting-state functional magnetic resonance imaging (fMRI) is a functional brain imaging method that is increasingly used to evaluate spontaneous brain activities in subjects at rest. ${ }^{11}$ Amplitude of low-frequency fluctuation (ALFF) is a resting-state fMRI analysis method used to measure spontaneous fluctuations in blood-oxygen level-dependent signal, which reflects regional spontaneous brain activities at rest. ALFF has been used to evaluate neurological conditions, including optic neuritis, ${ }^{12}$ glaucoma, ${ }^{13}$ and Parkinson's disease. ${ }^{14}$ This study aimed to evaluate spontaneous brain activities in acute OGI patients and evaluate its relationship with clinical features.

\section{Patients and methods}

\section{Patients}

A total of 18 acute OGI patients (16 males and two females, eight injured right eyes or eight injured left eyes) were recruited from the First Affiliated Hospital of South China University and the Ophthalmology Department of the First Affiliated Hospital of Nanchang University Hospital. The diagnostic criteria of acute OGI were 1) history of severe ocular trauma; 2) acute vision loss after ocular trauma; 3) corneal and scleral rupture; 4) low intraocular pressure; 5) orbital CT or orbital MRI showing incomplete eyeball wall; and 6) contralateral eye best-corrected vision $\geq 1.0$.

Exclusion criteria included 1) anterior segment diseases (such as glaucoma, cataract, strabismus, and pterygium) before acute eye injury; 2) other optic neuropathies (such as infection, inflammation, and ischemic disease, hereditary optic neuropathy, demyelinating disease, ischemic optic neuropathy, vascular lesions, toxic lesions, intraocular placeholder lesions, and conditions resulting in abnormal eye diseases); 3) mental nervous system disorders, diabetes, cardiovascular disease, and cerebral infarction disease such as systemic disorders; and 4) addictions (eg, drugs or alcohol).

A total of 18 healthy controls (HCs, 16 males and two females) with similar age, sex, and education status were recruited. All HCs met the following criteria: 1) no dysfunctions in brain in head MRI (cerebral hemorrhage, cerebral infarction, cerebrovascular malformation, and a brain tumor); 2) no eye disease; 3) normal mental nervous system; and 4) without MRI scanning contraindications (such as cardiac pacemaker, the body is equipped with a metal device, etc).

All research methods followed the Declaration of Helsinki and were approved by the Medical Ethics Committee of the First Affiliated Hospital of NanChang University. All subjects participated voluntarily and were notified of details and contents of the experiment, and all signed an informed consent form.

\section{MRI parameters}

MRI scanning was performed on a 3 T MR scanner (Trio; Siemens, Munich, Germany). All participants were required to be awake and quietly breathing until the end of the scan. Functional data were obtained with the following parameters: 176 images (repetition time $=1,900 \mathrm{~ms}$, echo time $=2.26 \mathrm{~ms}$, thickness $=1.0 \mathrm{~mm}$, gap $=0.5 \mathrm{~mm}$, acquisition matrix $=256 \times 256$, field of view $=250 \times 250 \mathrm{~mm}$, flip angle $=9^{\circ}$ ). We also obtained 240 functional images (repetition time $=2,000 \mathrm{~ms}$, echo time $=30 \mathrm{~ms}$, thickness $=4.0 \mathrm{~mm}$, gap $=1.2 \mathrm{~mm}$, acquisition matrix $=64 \times 64$, flip angle $=90^{\circ}$, field of view $=220 \times 220 \mathrm{~mm}, 29$ axial). The duration of the scanning was 15 minutes.

\section{fMRI data analysis}

A total of 240 functional data were classified using MRIcro software (www.MRIcro.com). The first ten time points were eliminated due to magnetization equilibration. And then, the rest of the data were preprocessed using the DPARSF (http://rfmri.org/DPARSF) software, as described previously. ${ }^{12,13}$

\section{A general linear model analysis}

A general linear model analysis was performed with the SPM8 toolkit (The MathWorks, Inc., Natick, MA, USA) to calculate the ALFF signal group differences in resting state between patients with OGI and HCs, after controlling for the effects of age and sex. The marked level was $P<0.05$.

\section{Brain-clinical features correlation analysis}

Brain areas with different ALFF values between groups were classified as regions of interest using REST software (Center for Cognition and Brain Disorders, HZNU., Hangzhou, Zhejiang, People's Republic of China). For each region of interest, the mean ALFF value was extracted by averaging the ALFF value. The relationship between the mean ALFF value in different regions of the OGI group and the clinical 
Table I Demographics and clinical measures by group

\begin{tabular}{lllll}
\hline Condition & OGI & HC & t & P-value $^{\text {a }}$ \\
\hline Male/female & $16 / 2$ & $16 / 2$ & N/A & $>0.99$ \\
Age (years) & $44.6 I \pm I 4.08$ & $46.00 \pm I 3.39$ & -0.303 & 0.764 \\
Weight $(\mathrm{kg})$ & $60.17 \pm 4.64$ & $60.22 \pm 4.00$ & -0.038 & 0.970 \\
Handedness & $18 \mathrm{R}$ & $\mathrm{I} R$ & $\mathrm{~N} / \mathrm{A}$ & $>0.99$ \\
Left or right injured eye & $8 / 8$ & $\mathrm{~N} / \mathrm{A}$ & $\mathrm{N} / \mathrm{A}$ & $\mathrm{N} / \mathrm{A}$ \\
Duration of OGI (hours) & $24.83 \pm 3 \mathrm{I} .07$ & $\mathrm{~N} / \mathrm{A}$ & $\mathrm{N} / \mathrm{A}$ & $\mathrm{N} / \mathrm{A}$ \\
Best-corrected VA-right & $0.57 \pm 0.58$ & $\mathrm{I} .17 \pm 0.20$ & -4.104 & $0.00 \mathrm{I}$ \\
Best-corrected VA-left & $0.64 \pm 0.53$ & $\mathrm{I} .12 \pm 0.17$ & -3.668 & $0.00 \mathrm{I}$ \\
\hline
\end{tabular}

Note: andependent $t$-tests comparing the two groups.

Abbreviations: OGI, open globe injury; $\mathrm{HC}$, healthy control; N/A, not applicable; VA, visual acuity.

features were calculated by bivariate correlation analysis. The marked difference was $P<0.05$.

\section{Clinical data analysis}

All clinical data of OGI patients were collected, including the duration of OGI and best-corrected visual acuity (VA) of both eyes.

\section{Results}

\section{Demographics and visual measurements}

The age of OGI patients and HCs was $44.61 \pm 14.08$ years

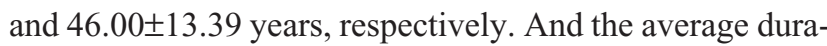
tion of OGI was $24.83 \pm 31.07$ hours. Meanwhile, there was no marked difference in weight $(P=0.970)$, age $(P=0.764)$, best-corrected VA-right ( $P=0.001)$, and best-corrected VAleft $(P=0.001)$ between the two groups (Table 1$)$.

\section{ALFF signal differences}

Compared with HCs, OGI patients had higher ALFF values in the left cuneus, left middle cingulum cortex, and bilateral precuneus (Figure 1 [red] and Table 2). The mean values of different ALFFs between the OGI group and HCs are displayed in Figure 2.

\section{Correlation analyses}

In the acute OGI group, we observed that the ALFF values of the bilateral precuneus showed a negative correlation with the duration of OGI ( $r=-0.493, P=0.038$, Figure $3 \mathrm{~A}$ ) and a negative correlation with vision of the injured eye $(r=0.583$, $P=0.011$, Figure $3 \mathrm{~B}$ ). The age of OGI patients was also negatively correlated with the ALFF signal value of the left middle cingulum cortex ( $r=-0.557, P=0.016$, Figure $3 C$ ) and negatively correlated with the mean ALFF signal value of the bilateral precuneus ( $r=-0.746, P<0.001$, Figure 3D).

\section{Receiver operating characteristic curve}

We proposed that ALFF differences between the OGI and HC groups might be useful diagnostic markers. The mean ALFF values of the different brain regions were used to analyze receiver operating characteristic curves. The area under the curve of the left middle cingulum cortex was 0.737 (Figure 4).

\section{Discussion}

To our knowledge, this is the first study to investigate the effect of acute OGI on brain activities using the ALFF

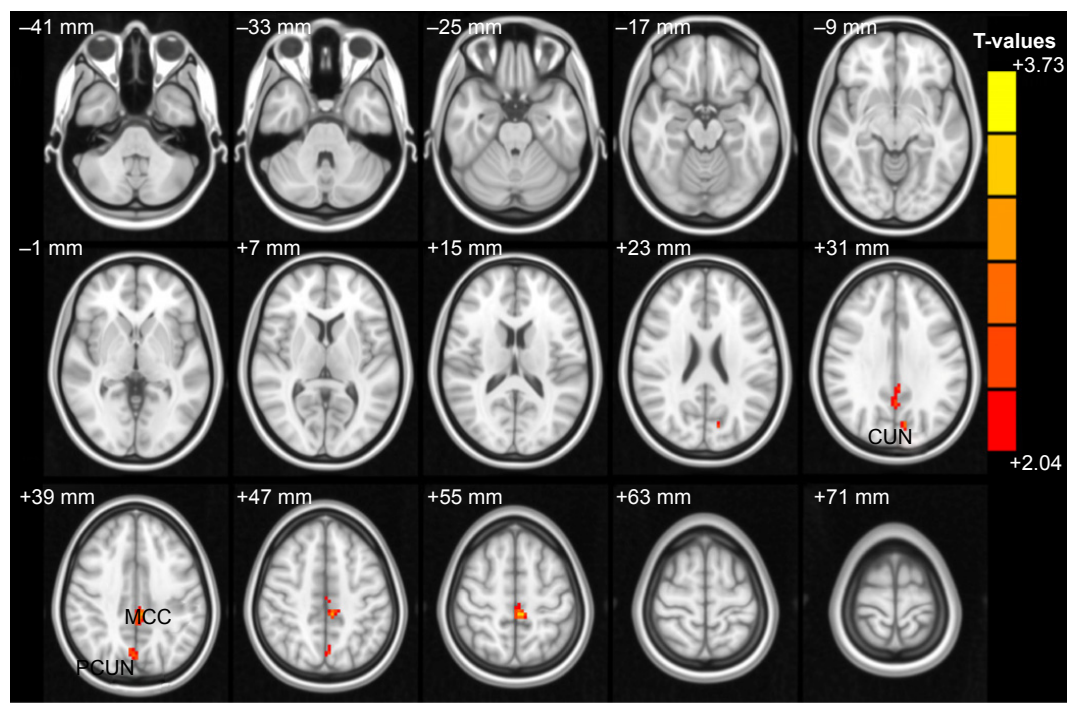

Figure I Spontaneous brain activity in the OGI and HC groups.

Notes: Significant differences in activity were observed for the left cuneus, left middle cingulum cortex, and bilateral precuneus. The red areas denote higher amplitude of low-frequency fluctuation values $P<0.05$ for multiple comparisons using Gaussian Random Field theory $(z>2.3$, cluster-wise $P<0.05$ corrected).

Abbreviations: OGI, open globe injury; HC, healthy controls; MCC, middle cingulum cortex; PCUN, precuneus. 
Table 2 Brain regions with significant ALFF differences between groups

\begin{tabular}{|c|c|c|c|c|c|c|c|}
\hline \multirow[t]{2}{*}{ ALFF } & \multicolumn{4}{|c|}{ OGI group and HCs group } & \multicolumn{3}{|c|}{$\begin{array}{l}\text { MNI } \\
\text { coordinates }\end{array}$} \\
\hline & Brain areas & BA & T-values & $\begin{array}{l}\text { Peak } \\
\text { voxels }\end{array}$ & $x$ & $y$ & $\mathbf{z}$ \\
\hline \multicolumn{8}{|c|}{$\mathrm{OGI}>\mathrm{HC}$} \\
\hline I & Left cuneus & 7 & 2.631 & 16 & -9 & -75 & 30 \\
\hline 2 & $\begin{array}{l}\text { Left middle cingulum } \\
\text { cortex }\end{array}$ & 31 & 3.731 & 125 & -3 & -36 & 36 \\
\hline 3 & Bilateral precuneus & 7 & 2.663 & 37 & 3 & -72 & 39 \\
\hline
\end{tabular}

Note: The statistical threshold was set at voxel with $P<0.05$ for multiple comparisons using Gaussian Random Field theory ( $z>2.3$, cluster-wise $P<0.05$ corrected).

Abbreviations: ALFF, amplitude of low-frequency fluctuation; OGI, open globe injury; HCs, healthy controls; MNI, Montreal Neurological Institute; BA, Brodmann area.

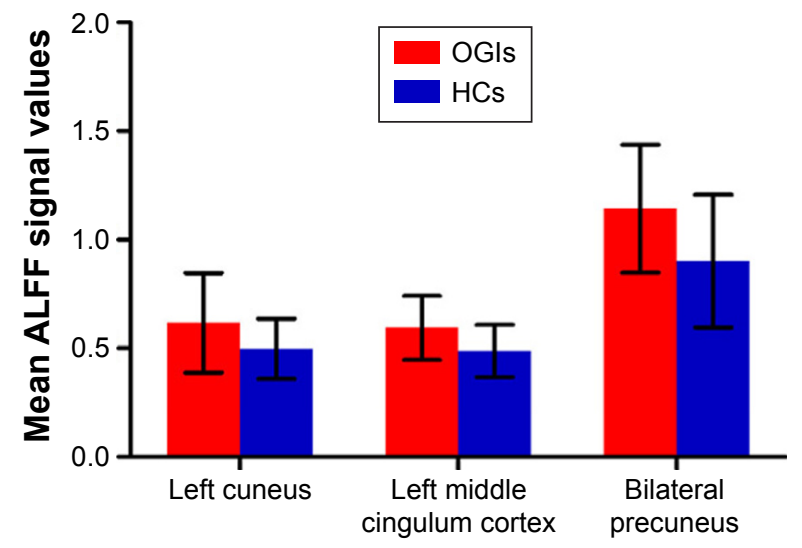

Altered ALFF regions

Figure 2 The mean values of altered ALFF between the OGI and $\mathrm{HC}$ groups. Note: Data presented as mean \pm standard deviation.

Abbreviations: ALFF, amplitude of low-frequency fluctuation; OGls, open globe injuries; HCs, healthy controls.

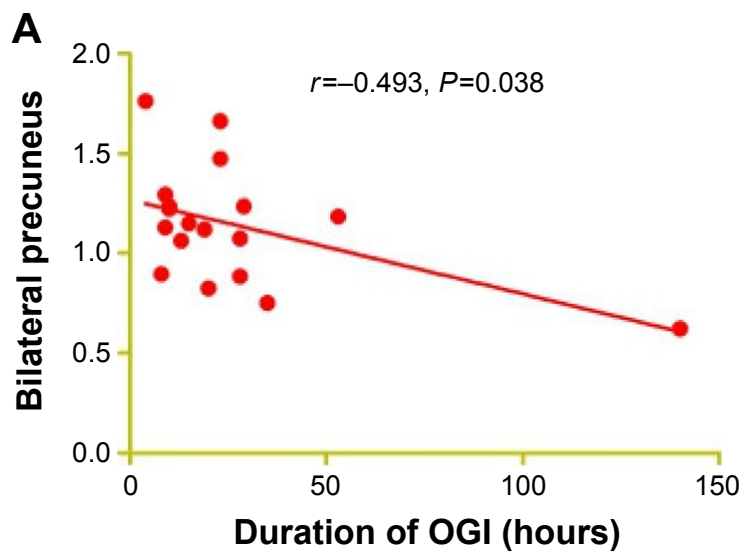

Figure 3 (Continued) technique. Acute OGI patients had significantly higher ALFF signal values in the left cuneus, left middle cingulum cortex, and bilateral precuneus. Furthermore, we found that the age of OGI patients showed a negative correlation with the ALFF value of the left middle cingulum cortex $(r=-0.557$, $P=0.016)$ and a negative correlation with the mean ALFF signal value of the bilateral precuneus $(r=-0.746, P<0.001)$. The ALFF signal value of the bilateral precuneus showed a negative correlation with the duration of OGI $(r=-0.493$, $P=0.038$ ) and a positive correlation with vision of the injured eye $(r=0.583, P=0.011)$.

The precuneus located on forward of the cuneus is involved in many functions, including visuospatial imagery, ${ }^{15}$ attention, ${ }^{16}$ episodic memory, ${ }^{17}$ and default-mode network. ${ }^{18}$ A previous study showed that the precuneus played an important role in encoding spatial locations. ${ }^{19}$ In addition, the precuneus was also involved in motor image, which transfers the motor signal to supplementary motor area. ${ }^{20}$ Meanwhile, the precuneus was in charge of spatial working memory. ${ }^{21}$ In our study, we observed that patients with acute OGI showed higher ALFF in bilateral precuneus, which may reflect the dysfunction of visuospatial imagery in OGI patients. Moreover, we observed that the ALFF value of the bilateral precuneus showed a negative correlation with the duration of OGI $(r=-0.493$, $P=0.038$ ). We speculated that there were significant brain activities in the bilateral precuneus in early phase of acute OGI. Furthermore, we also found that the ALFF values in bilateral precuneus showed a positive correlation with the vision of the injured eye $(r=0.583, P=0.011)$. This means that the severity of OGI and the precuneus function were closely related. We speculated that OGI might lead to dysfunction of the precuneus.

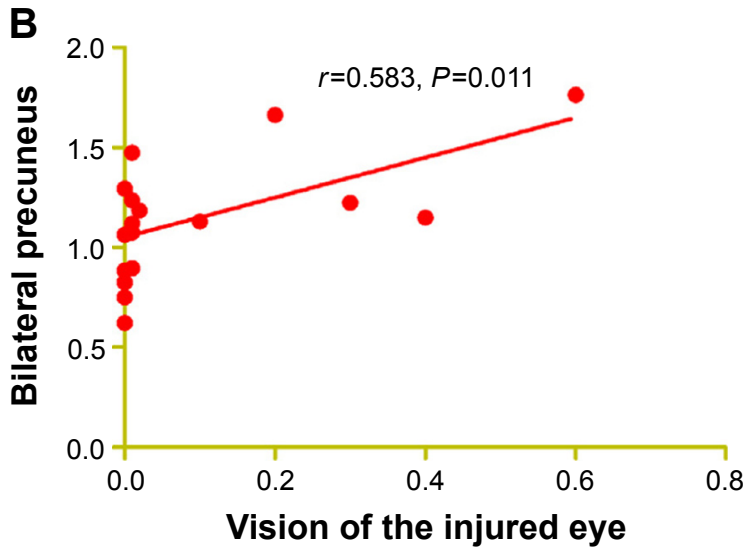



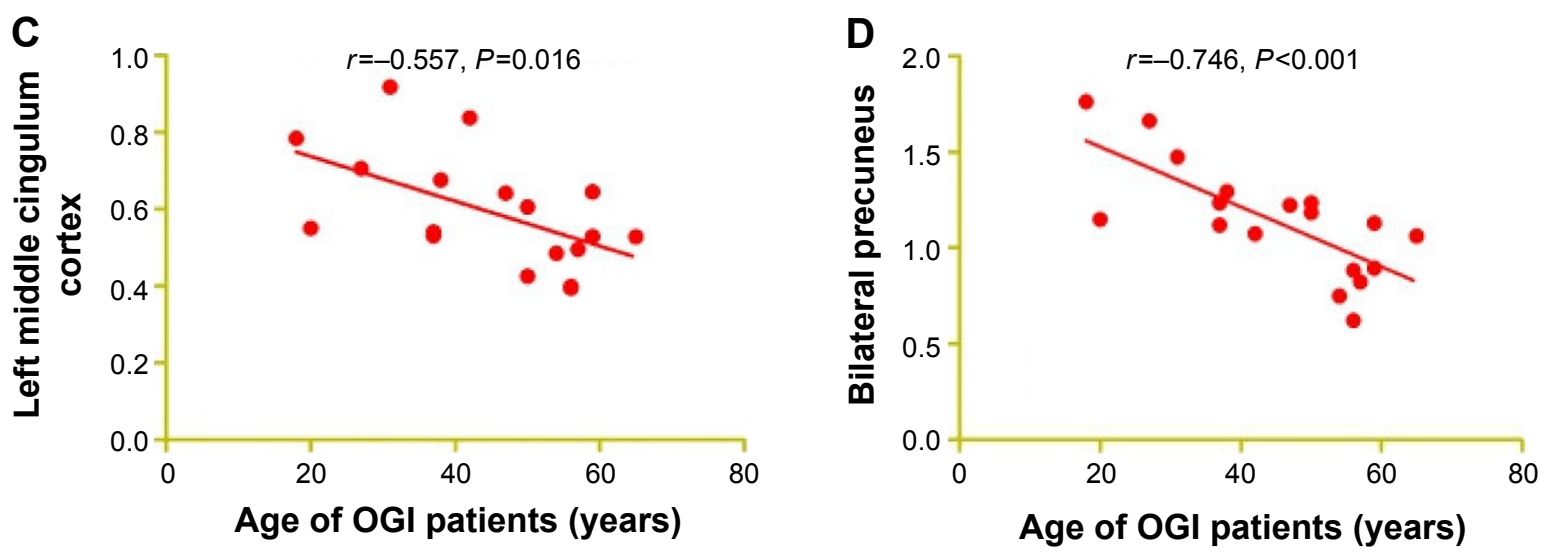

Figure 3 Correlations between mean ALFF signal values and behavioral performance.

Notes: The ALFF signal value of the bilateral precuneus showed a negative correlation with the duration of $O G I(r=-0.493, P=0.038 ; \mathbf{A})$ and a negative correlation with vision of the injured eye $(r=0.583, P=0.01$ I; B). The age of OGI patients showed a negative correlation with the ALFF signal value of the left middle cingulum cortex ( $r=-0.557$, $P=0.016 ; \mathbf{C})$ and a negative correlation with the mean ALFF signal value of the bilateral precuneus $(r=-0.746, P<0.001 ; \mathbf{D})$.

Abbreviations: ALFF, amplitude of low-frequency fluctuation; OGI, open globe injury.

The cuneus located in the occipital lobe of the brain is involved in visual processing. The occipital lobe is the visual processing center, which contains the visual cortex. A previous study reported that the cuneus modifies visual information to extrastriate cortices via primary visual cortex V1.22 In this study, we observed that acute OGI patients showed higher ALFF in the left cuneus. We speculated that OGI might lead to the dysfunction of the cuneus.

Surrounding the whole brain, the cingulum is located beneath the cingulate gyrus. As a part of the limbic system, the cingulum is involved in many functions such as memory, ${ }^{23}$ cognitive functions,${ }^{24}$ and attention. ${ }^{25}$ In our study, we observed that acute OGI patients showed higher ALFF

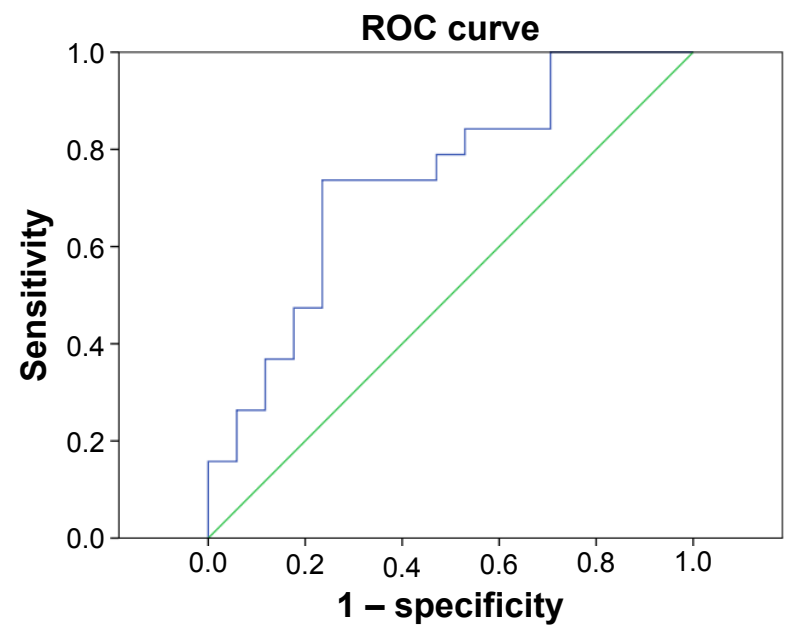

Figure 4 ROC curve analysis of mean ALFF signal values for left middle cingulum cortex brain regions.

Note: The area under the ROC curve for ALFF values was 0.737 ( $P=0.015$; 95\% confidence interval: 0.572-0.902).

Abbreviations: ROC, receiver operating characteristic; ALFF, amplitude of lowfrequency fluctuation. in the left middle cingulum cortex, which may reflect the dysfunction of left middle cingulum cortex caused by OGI. Besides, we speculated that OGI may lead to the dysfunction of limbic system. Furthermore, we observed that the age of OGI patients showed a negative correlation with the ALFF value of the left middle cingulum cortex. This means that the dysfunction of the limbic system may be related to the age of OGI patients.

\section{Conclusion}

This study demonstrated that acute OGI patients had dysfunction of brain activities. These findings may provide important information to explain the underlying neural mechanisms of OGI, which are beneficial to clinical diagnosis.

\section{Acknowledgments}

This study was supported by the National Natural Science Foundation of China (81160118, 81100648, and 81400372); Jiangxi Province Voyage Project (2014022); Youth Science Foundation of Jiangxi Province (20151BAB215016); Technology and Science Foundation of Jiangxi Province (20151BBG70223); and Health Development Planning Commission Science Foundation of Jiangxi Province (20155154). This was not an industry-supported study.

\section{Disclosure}

The authors report no conflicts of interest in this work.

\section{References}

1. Cillino S, Casuccio A, Di Pace F, Pillitteri F, Cillino G. A five-year retrospective study of the epidemiological characteristics and visual outcomes of patients hospitalized for ocular trauma in a Mediterranean area. BMC Ophthalmol. 2008;8:6. 
2. Pandita A, Merriman M. Ocular trauma epidemiology: 10-year retrospective study. N Z Med J. 2012;125(1348):61-69.

3. Nawani N, Vazirani J, Ojha H, Sangwan VS. Conjunctival pedicle flap in management of open globe injury with corneal tissue loss. BMJ Case Rep. 2016;2016:bcr2015213703.

4. Smith MP, Colyer MH, Weichel ED, Stutzman RD. Traumatic cataracts secondary to combat ocular trauma. J Cataract Refract Surg. 2015;41(8): 1693-1698.

5. Nuzzi R, Buschini E, Actis AG. Ophthalmic evaluation and management of traumatic accidents associated with retinal breaks and detachment: a retrospective study. Eur J Ophthalmol. 2012;22(4):641-646.

6. Lipke KJ, Gümbel HO. Emergency treatment of ocular trauma. Facial Plast Surg. 2015;31(4):345-350.

7. Andreoli MT, Yiu G, Hart L, Andreoli CM. B-scan ultrasonography following open globe repair. Eye (Lond). 2014;28(4):381-385.

8. Arey ML, Mootha VV, Whittemore AR, Chason DP, Blomquist PH. Computed tomography in the diagnosis of occult open-globe injuries. Ophthalmology. 2007;114(8):1448-1452.

9. Hoffstetter P, Schreyer AG, Schreyer CI, et al. Multidetector CT (MD-CT) in the diagnosis of uncertain open globe injuries. Rofo. 2010; 182(2):151-154.

10. Rao SK, Nunez D, Gahbauer H. MRI evaluation of an open globe injury. Emerg Radiol. 2003;10(3):144-146.

11. Biswal BB. Resting state fMRI: a personal history. Neuroimage. 2012; 62(2):938-944.

12. Huang X, Cai FQ, Hu PH, et al. Disturbed spontaneous brainactivity pattern in patients with optic neuritis using amplitude of low-frequency fluctuation: a functional magnetic resonance imaging study. Neuropsychiatr Dis Treat. 2015;11:3075-3083.

13. Huang X, Zhong YL, Zeng XJ, et al. Disturbed spontaneous brain activity pattern in patients with primary angle-closure glaucoma using amplitude of low-frequency fluctuation: a fMRI study. Neuropsychiatr Dis Treat. 2015;11:1877-1883.

14. Kwak Y, Peltier SJ, Bohnen NI, Müller ML, Dayalu P, Seidler RD. L-DOPA changes spontaneous low-frequency BOLD signal oscillations in Parkinson's disease: a resting state fMRI study. Front Syst Neurosci. 2012;6:52
15. Cavanna AE, Trimble MR. The precuneus: a review of its functional anatomy and behavioural correlates. Brain. 2006;129(pt 3):564-583.

16. Nagahama Y, Okada T, Katsumi $Y$, et al. Transient neural activity in the medial superior frontal gyrus and precuneus time locked with attention shift between object features. Neuroimage. 1999;10(2):193-199.

17. Lundstrom BN, Ingvar M, Petersson KM. The role of precuneus and left inferior frontal cortex during source memory episodic retrieval. Neuroimage. 2005;27(4):824-834.

18. Utevsky AV, Smith DV, Huettel SA. Precuneus is a functional core of the default-mode network. J Neurosci. 2014;34(3):932-940.

19. Frings L, Wagner K, Quiske A, et al. Precuneus is involved in allocentric spatial location encoding and recognition. Exp Brain Res. 2006;173(4): 661-672.

20. Ogiso T, Kobayashi K, Sugishita M. The precuneus in motor imagery: a magnetoencephalographic study. Neuroreport. 2000;11(6): $1345-1349$

21. Wallentin M, Weed E, Østergaard L, Mouridsen K, Roepstorff A. Accessing the mental space-Spatial working memory processes for language and vision overlap in precuneus. Hum Brain Mapp. 2008;29(5): 524-532.

22. Vanni S, Tanskanen T, Seppä M, Uutela K, Hari R. Coinciding early activation of the human primary visual cortex and anteromedial cuneus. Proc Natl Acad Sci U S A. 2001;98(5):2776-2780.

23. Delano-Wood L, Stricker NH, Sorg SF, et al. Posterior cingulum white matter disruption and its associations with verbal memory and stroke risk in mild cognitive impairment. J Alzheimers Dis. 2012;29(3): 589-603.

24. Lin YC, Shih YC, Tseng WY, et al. Cingulum correlates of cognitive functions in patients with mild cognitive impairment and early Alzheimer's disease: a diffusion spectrum imaging study. Brain Topogr. 2014;27(3):393-402.

25. Takahashi M, Iwamoto K, Fukatsu H, Naganawa S, Iidaka T, Ozaki N. White matter microstructure of the cingulum and cerebellar peduncle is related to sustained attention and working memory: a diffusion tensor imaging study. Neurosci Lett. 2010;477(2):72-76.
Neuropsychiatric Disease and Treatment

\section{Publish your work in this journal}

Neuropsychiatric Disease and Treatment is an international, peerreviewed journal of clinical therapeutics and pharmacology focusing on concise rapid reporting of clinical or pre-clinical studies on a range of neuropsychiatric and neurological disorders. This journal is indexed on PubMed Central, the 'PsycINFO' database and CAS,

\section{Dovepress}

and is the official journal of The International Neuropsychiatric Association (INA). The manuscript management system is completely online and includes a very quick and fair peer-review system, which is all easy to use. Visit http://www.dovepress.com/testimonials.php to read real quotes from published authors. 